\title{
A Study of Touchless Fingerprint Recognition System
}

\author{
Chulhan Lee, Sanghoon Lee, and Jaihie Kim \\ Department of Electrical and Electronic Engineering, Yonsei University, \\ Biometrics Engineering Research Center (BERC), \\ Republic of Korea \\ devices@yonsei.ac.kr
}

\begin{abstract}
Fingerprint recognition systems are widely used in the field of biometrics. Many existing fingerprint sensors acquire fingerprint images as the user's fingerprint is contacted on a solid flat sensor. Because of this contact, input images from the same finger can be quite different and there are latent fingerprint issues that can lead to forgery and hygienic problems. For these reasons, a touchless fingerprint recognition system has been investigated, in which a fingerprint image can be captured without contact. While this system can solve the problems which arise through contact of the user's finger, other challenges emerge, for example, low ridge-valley contrast, and $3 \mathrm{D}$ to $2 \mathrm{D}$ image mapping. In this paper we discuss both the disadvantages and the advantages of touchless fingerprint systems and introduce the hardware and algorithm approach to solve the problems. We describe the structure of illuminator and the wavelength of light to acquire a high contrast fingerprint images. To solve the problem of $3 \mathrm{D}$ to $2 \mathrm{D}$ image mapping, we describe the method to remove the strong view difference fingerprint images. Experiments show that the touchless fingerprint system has better performance than the conventional touch based system.
\end{abstract}

\section{Introduction}

Many biometric features have been used to confirm the identity of a given human. Some of these recognition features have included iris, face, fingerprint, voice, hand geometry, and the retinal pattern of eyes. Among all these features, fingerprint recognition has been the most popular and reliable biometric feature for automatic personal identification. Various types of sensors (including optical, thermal, and capacitive sensors) have been developed in order to acquire good fingerprint images with appropriate characteristics. Also, a large variety of algorithms have been proposed in order to achieve better authentication performance. In spite of all these efforts to acquire good fingerprint images and enhance performance, there are a number of problems which occur when using conventional touch-based sensors.

\subsection{Problems with Touch-Based Sensors}

In order to acquire fingerprint images with conventional touch-based sensors, the user must place his finger on the flat window of the sensor. Because the skin 


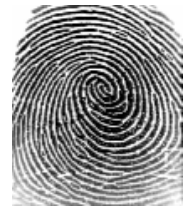

(a)

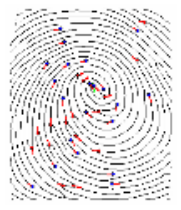

(b)

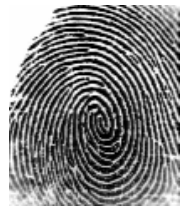

(c)

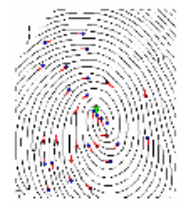

(d)

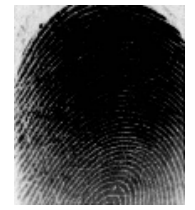

(e)

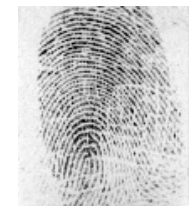

(f)

Fig. 1. Distorted images from a touch-based sensor. (a) and (c) are gray-value images, (b) and (c) are corresponding minutiae extracted images, and (e) and (f) show the effects of different strengths of impression.

of the finger is not flat, the user must apply enough pressure on the window to obtain sufficient size and achieve good image quality. However, this pressure produces unavoidable physical distortion in arbitrary directions, which is represented differently throughout every area of the same fingerprint image. Also, since the image varies with each impression, each fingerprint image from the same finger can appear quite different. Fig. 1 shows the images from touchbased sensor. Because of different pressure, the relative position and types of corresponding minutiae are different (Fig. 1(a)-(d)). Also, the sizes of the fingerprint areas and the quality of the fingerprint images are quite different (Fig. 1(e),(f)). These problems critically affect fingerprint recognition. There are also latent fingerprint problems. A latent fingerprint refers to the trail of the fingerprint on the window of the sensor. This can lead to hygienic problems as well as fraudulent use, such as the faking of fingerprints [1. The issue of protecting biometric information and privacy is paramount in biometric technology. As mentioned above, previous touch-based sensor approaches can lead to several problems. To solve these problems, [2 proposed the elastic minutiae matching algorithm using the TPS (thin-plate spline) model. In this method, corresponding points were detected using local minutiae information, and global transformation was determined with the TPS model. Although the method produced higher matching scores when compared to the ridge-matching algorithm, it requires a good minutiae extraction algorithm that extracts well in even distorted images. However, if a fingerprint image is highly distorted, to extract usable minutiae is very difficult. 3] proposed detection of artificial fingers. This method showed that detection of a perspiration pattern in a time progression of fingerprint images can be used as an anti-spoofing measure. However, environmental factors (such as temperature and humidity) and user-specific factors (such as skin humidity and pressure) are not taken into account. In this paper, we investigate a touchless fingerprint system that fundamentally overcomes the problems involved in conventional touch-based sensors. This paper is organized as follows. In section 2, we explain the advantages and disadvantages of touchless fingerprint systems and introduce the hardware approach and the algorithm approach to address the disadvantages. In section 3, a comparison between touch-based sensors and touchless sensors is given in terms of recognition performance. Finally, conclusions and suggestions for future works are discussed in section 4 . 


\section{Touchless Fingerprint System}

In order to solve the innate problems of touch-based sensors, we studied a touchless fingerprint recognition system. [4] developed a touchless fingerprint sensor using a camera. They used a polarizer filter and a band-pass filter in order to acquire a good quality image. Unfortunately, there is no explanation between the illuminator and filters which affected image quality. We also used a camera to capture fingerprint images in our touchless fingerprint sensor. Advantages of using a camera include: i) the fingerprint image can be acquired without plastic distortion from contact pressure, ii) latent fingerprints do not appear on the sensor, iii) hygienic problems are reduced, and iv) a large image area can be captured quickly. The combination of distortion-free fingerprint images and large image areas is desirable in order to acquire many minutiae in the same relative location and direction at every instance. Therefore, this combination helps the authentication system to have low FAR and FRR. While there are strong advantages to this system, there are also new disadvantages. These can be classified into two areas: low contrast between the ridges and valleys, and 3D to $2 \mathrm{D}$ image mapping. Low ridge-valley contrast is caused by the motion blur of hand tremble, camera background noise, and a small Dof (Depth of field). Because the 3-dimensional object finger is projected onto a 2-dimensional image plane, the difference of the camera viewpoint caused by the 3 -dimensional rotation of the finger can produce a small common area between the enrolled and input images. This leads to an increased false acceptance rate (FRR). Fig. 2 shows the sample images which represent the above-mentioned problems. In this paper, we propose the hardware approach in order to solve the low ridge-valley contrast and the algorithm approach in order to solve the difference between the camera viewpoints.

\subsection{The Hardware Approach: Low Ridge-Valley Contrast Issue}

Structure of Device. Motion blur generally stems from the long capture time relative to the slight motion of the finger. Although high-sensitivity sensors can handle fast shutter speed, images normally contain increased electrical background noise. Although a large aperture stop could be an alternative way to retain rapid shutter speed, this method is an unsuitable way to guarantee the required Dof, which is important to keep the variation range of the finger

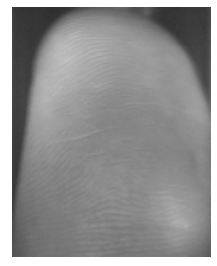

(a)

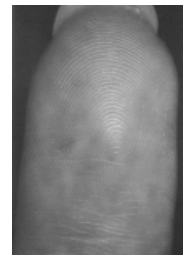

(b)

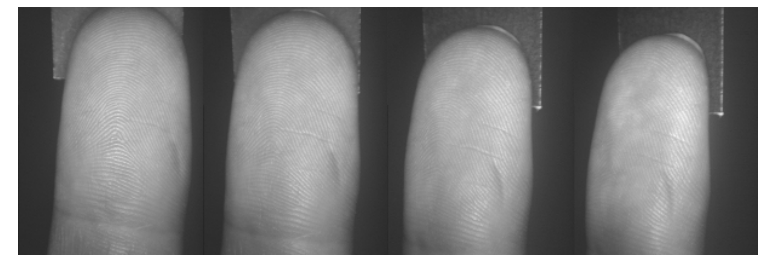

(c)

Fig. 2. (a) Motion-blurred image, (b) partially defocusing, (c) images from rolled fingers 


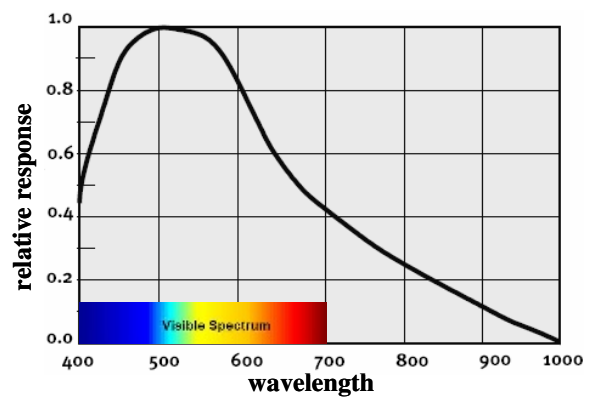

Fig. 3. The spectral sensitivities curve of the camera

position in focus. Hence, a small apparatus and appropriate lighting gadgetry are necessary. For this reason, we adopt a finger support to decrease motion blur and an appropriate illuminator to obtain uniform lighting and sufficient brightness. There are several types of illuminator structures which can be used to obtain good images in special applications [5]. For example, dark-field lights illuminate objects from their sides with specific patterns to emphasize shadows and enhance image quality. A ring-type illuminator, especially, is easy to build up and produces uniform illumination on the object, and on-axis lights illuminate the object relative to the camera axis. Among those types, the ring-type and their modified types simply and stably obtain uniform light conditions.

Wavelengths of Illuminators. To acquire good quality fingerprint images for fingerprint recognition, both the spectral sensitivities of the camera and the skin reflectance of the finger must be considered. The method proposed in [6] measured the light reflected from the skin using a high-resolution, high-accuracy spectrograph under precisely calibrated illumination conditions. This showed that the skin reflectance of humans is mainly influenced by both melanin and hemoglobin. However, the skin reflectance of the palm (including the fingers) is mainly influenced by oxygenated hemoglobin because of the scarcity of melanin in the palm. In this paper, the hemoglobin absorption spectrum was founded. The ratio of hemoglobin absorption is lower around 500nm and higher around $545 \mathrm{~nm}$ and $575 \mathrm{~nm}$. Because it is desirable to observe only the surface of the finger and remove the effect of hemoglobin in order to obtain a high-contrast fingerprint image, the wavelength at lower hemoglobin absorption must be chosen on the wavelength of the illuminator of the touchless fingerprint sensor. Fig. 3 shows the spectral sensitivities of the normal CCD. The sensitivities are high around 500nm. Considering both the skin reflectance of the finger and the spectral sensitivities of each camera, the wavelength of light on the touchless fingerprint sensor can be determined.

\subsection{Software Approach: 3D to 2D Image Mapping Issue}

Since touchless fingerprint sensors acquire 2-dimensional fingerprint images from 3-dimensional finger skins, a significant view difference is generated when rolling 
the finger in the image acquisition step. We divided the view difference image into a weak view difference image and a strong view difference image. The weak view difference image usually has a small influence on the fingerprint and is allowable through elastic matching by the tolerance parameters of the bounding box 7 . When the strong view difference image appears, ridges on the slanted skin are densified on the image (due to the $3 \mathrm{D}$ to $2 \mathrm{D}$ projections), they are apt to have type-changed, missed or even false minutiae. Moreover, the foreground becomes the near-boundary region and also becomes dark or out of focus. This decreases the number of usable minutiae as well as the good quality foreground, and also reduces the common area between the fingerprint impressions, which results in bad system performance. Therefore, it is desirable to reject images with strong view differences and instruct the user to retry the input. To determine the strong view difference image, we measure the distance between the core and the center axis of the finger. Fig. 4 shows the distance as determined by the rolled finger. The larger the distance between the core and the center axis, the more the image is rolled. Most fingerprints have at least one core (except fingerprints of the arch type). Though these fingerprints have no core point, we can detect one singular point that is invariant to rolling. We implemented the core point detection algorithm using [8. This method is an accumulating mechanism similar to a Hough transform. Using a ridge orientation map, this algorithm follows a path that is perpendicular to the ridge orientation and accumulates a histogram. After finishing all searches, the location of the core point can be determined by

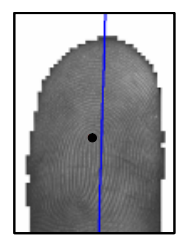

(a)

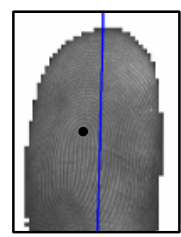

(b)

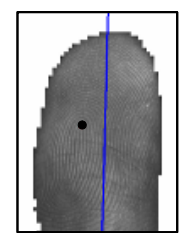

(c)

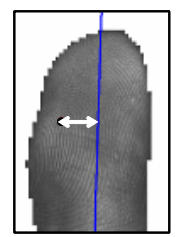

(d)

Fig. 4. The distance between the core and center axis of rolled finger. (a) 0 degree, (b) 10 degrees, (c) 20 degrees, (d) 40 degrees.

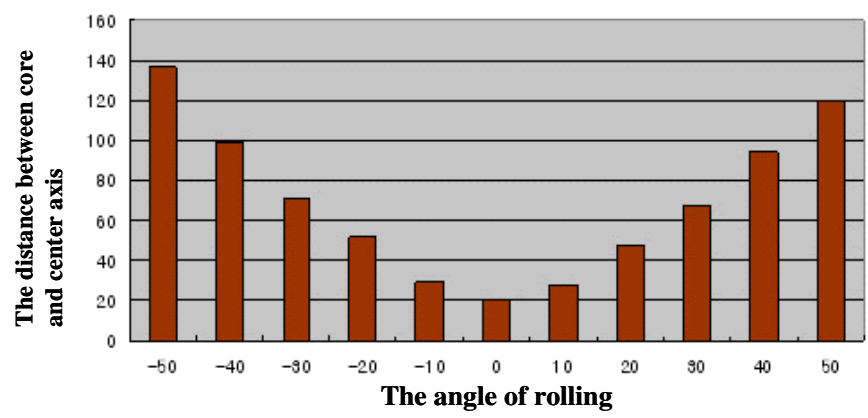

Fig. 5. The distance between the core and center axis of rolled finger 
detecting the maximum position on the histogram. This method is robust to noise and can detect the maximum position for the specific arch type. The center axis of the finger is determined as the $\mathrm{KL}$ (Karhunen and Loeve) transform with the segmented image. The distance that determines the strong view difference image can be defined as follows:

$$
\text { Distance }=\frac{\left|a x_{c}+b y_{c}+c\right|}{\sqrt{a^{2}+b^{2}}}
$$

where $\left(x_{c}, y_{c}\right)$ is the location of the core point and $\mathrm{a}, \mathrm{b}$ and $\mathrm{c}$ are the coefficients of the center axis $a x+b y+c=0$. Fig. 5 shows the averaged distance of 50 fingers according to the sampled rolling angle. We can observe that the distance can discriminate between the strong view difference image and the weak view difference image.

\section{Experimental Results}

\subsection{Comparison Between Touch-Based Sensors and Touchless Sensors}

For this comparison, we collected 1630 fingerprint images from 163 fingers using a touchless-sensor and also a touch-based optical sensor (Ditgen FD1000 [9]). To avoid strong rolled fingerprint images, we mentioned this to the users

Table 1. The specification of sensors

\begin{tabular}{|c|c|c|}
\hline & Touchless sensor & Touch-based sensor \\
\hline \hline Size of input window & $24 \mathrm{~mm} \times 34 \mathrm{~mm}$ & $17 \mathrm{~mm} \times 19 \mathrm{~mm}$ \\
\hline Resolution & $450 \mathrm{dpi}$ & $500 \mathrm{dpi}$ \\
\hline Size of image & $480 \times 640$ pixels (Fingerprint $320 \times 450$ Pixels) & $280 \times 320$ pixels \\
\hline
\end{tabular}

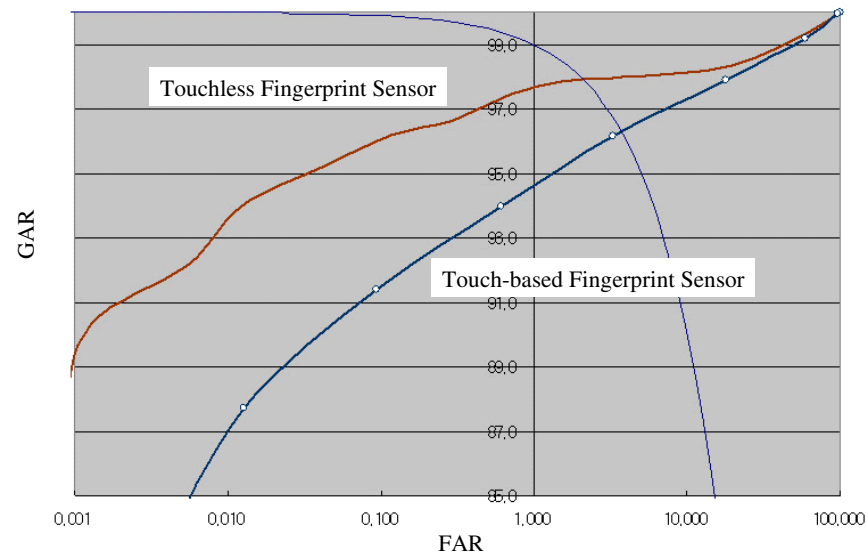

Fig. 6. The ROC of touch-based fingerprint recognition and touchless fingerprint recognition 


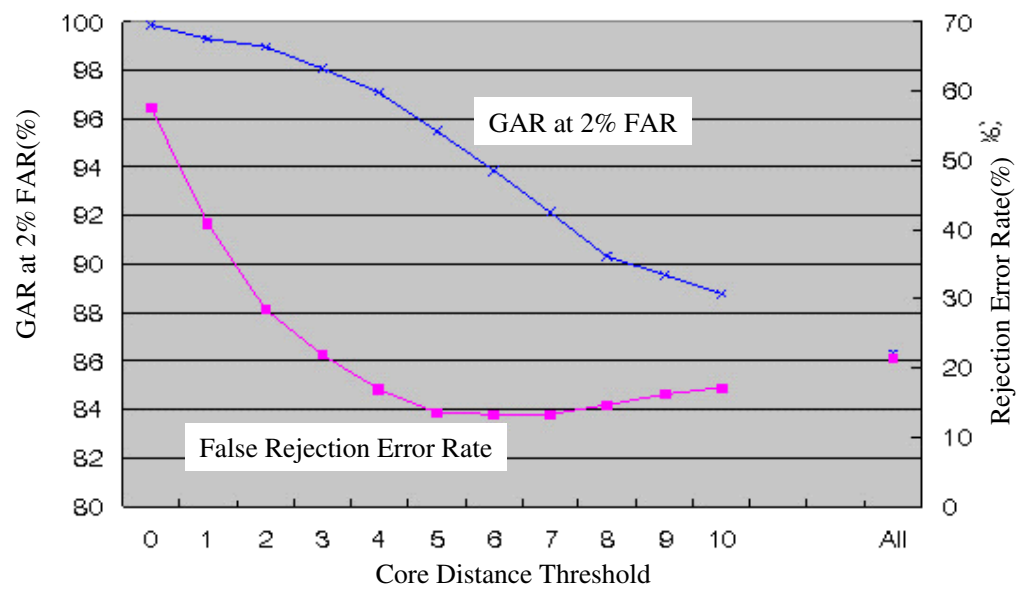

Fig. 7. Performance of the system (GAR at $2 \%$ FAR) and the false reject error rate

before acquiring the images. Table 1 shows the specifications of the two sensors. After acquiring the images, we adopted the same fingerprint image processing algorithm [10] and matching algorithm [11]. Fig. 6 shows the ROC of the two results. From these experimental results, we can observe that the performance of touchless fingerprint sensors is better than the performance of touch-based fingerprint sensors.

\subsection{Rejecting the Strong View Difference Image: An Evaluation of the Method}

1100 rolled finger images were collected from 50 fingers. Each finger was rolled from -50 degrees to +50 degrees with an interval of 10 degrees. Fig 7 shows the relationship between the performance of the system (GAR at $2 \%$ FAR) and the false reject error rate. The false reject error is composed of two types of errors. The type-I error is the number of reject images corresponding to the weak view difference image. The type-II error is the number of acceptance image corresponding to the strong view difference image. We defined the strong and weak view difference images by matching the algorithms with the matching threshold T. The matching threshold is defined by experiment 3.1 .

\section{Conclusions and Future Works}

In fingerprint recognition, conventional touch-based sensors contain innate problems that are caused by the contact of the finger. Some examples of these problems are distortions in the fingerprint images, latent fingerprints and hygienic problems. To overcome these fundamental problems, we investigated a touchless fingerprint recognition system. While we obtained a large distortion-free fingerprint image, new problems needed to be dealt with, such as low contrast between ridges and 
valleys and $3 \mathrm{D}$ to $2 \mathrm{D}$ image mapping. To acquire good fingerprint images, we introduced a hardware approach that used the structure of the device and the wavelengths of light. Also, we proposed a strong view difference image rejection method using the distance between the core and the center axis of the finger in order to overcome the $3 \mathrm{D}$ to $2 \mathrm{D}$ image mapping problem. In the experiments, we compared the touchless fingerprint sensor with the touch-based fingerprint sensor in terms of performance and evaluated the proposed rejection method.

In future work, we will develop the matching algorithm that is invariant to $3 \mathrm{D}$ camera viewpoint change and compare fingerprint recognition system in images captured with touchless sensors and other touch-based sensors. In this comparison, we will compare not only verification performance but also image quality, the convenience of usage, the size of fingerprint image, and the number of true minutiae.

\section{Acknowledgements}

This work was supported by Korea Science and Engineering Foundation (KOSEF) through the Biometrics Engineering Research Center at Yonsei University.

\section{References}

1. T. Matsumoto, H. Matsumoto, K. Yamada, and S. Hoshino, "Impact of artificial "gummy" fingers on fingerprint systems", In Proceedings of SPIE Vol. Num.4677, Jan 2002.

2. Bazen, A.M. and Gerez, S.H., "Elastic minutiae matching by means of thin-plate spline models" Pattern Recognition, 2002. Proceedings. 16th International Conference on Vol. 2, pp. 985 - 988, Aug. 2002.

3. Derakhshani R., Schuckers S. A. C., Hornak L., and O'Gorman L. "Determination of vitality from a non-invasive biomedical measurement for use in fingerprint scanners." Pattern Recognition Journal, Vol.36, (2003) 383-396.

4. Hans J. Einighammer and Jens Elnighammer, "System for the Touchless Recognition of Hand and Finger Line", US6404904, 2002.

5. Pantazis Mouroulis, "Visual Instrumentation: Optical Design \& Engineering Principles" McGraw-Hill Professional, 1999.

6. Angelopoulou Elli, "Understanding the Color of Human Skin. ", Proceedings of the 2001 SPIE conference on Human Vision and Electronic Imaging VI, SPIE Vol. 4299, pp. 243-251, May 2001.

7. Jain A., Lin H. and Bolle R., "On-line Fingerprint Verification," IEEE Trans. on Pattern Analysis and Machine Intelligence, Vol. 19, no. 4, pp.302-314, Apr. 1997.

8. Huvanandana, S., Changick Kim and Jenq-Neng Hwang, "Reliable and fast fingerprint identification for security applications", IEEE International Conference on Image Processing, vol. II, pp. 503-506, Vancouver, Canada, October 2000.

9. http://www.digent.co.kr

10. L. Hong, Y. Wan and A.K. Jain, "Fingerprint Image Enhancement: Algorithms and Performance Evaluation", IEEE Transactions on PAMI, Vol. 20, No. 8, pp.777-789, August 1998.

11. D. Lee, K. Choi and Jaihie Kim, "A Robust Fingerprint Matching Algorithm Using Local Alignment", International Conference on Pattern Recognition, Quebec, Canada, August 2002. 